\title{
The effectiveness of chemical denture cleansers and ultrasonic device in biofilm removal from complete dentures
}

Patrícia Costa CRUZ ${ }^{1}$, Ingrid Machado de ANDRADE ${ }^{1}$, Amanda PERACINI ${ }^{1}$, Maria Cristina Monteiro de SOUZAGUGELMIN ${ }^{2}$, Cláudia Helena SILVA-LOVATO ${ }^{3}$, Raphael Freitas de SOUZA ${ }^{4}$, Helena de Freitas Oliveira PARANHOS ${ }^{5}$

\author{
1- DDS, MSc, graduate student, Department of Dental Materials and Prosthodontics, Ribeirão Preto Dental School, University of São Paulo, Ribeirão Preto, \\ SP, Brazil. \\ 2- DDS, MSc, PhD, Associate Professor, Department of Clinical, Toxicological and Bromatological Analysis, Faculty of Pharmaceutical Sciences of Ribeirão \\ Preto, University of São Paulo, Ribeirão Preto, SP, Brazil. \\ 3- DDS, MSc, PhD, Associate Professor, Department of Dental Materials and Prosthodontics, Ribeirão Preto Dental School, University of São Paulo, Ribeirão \\ Preto, SP, Brazil. \\ 4- DDS, MSc, PhD, Assistent Professor, Department of Dental Materials and Prosthodontics, Ribeirão Preto Dental School, University of São Paulo, Ribeirão \\ Preto, SP, Brazil. \\ 5- Titular Professor, Department of Dental Materials and Prosthodontics, Ribeirão Preto Dental School, University of São Paulo, Ribeirão Preto, SP, Brazil.
}

Corresponding address: Helena de Freitas Oliveira Paranhos - Avenida do café, s/n - Ribeirão Preto - SP - Brasil - 14049-904 - e-mail: helenpar@forp.usp.br

Received: September 28, 2009 - Modification: April 30, 2010 - Accepted: May 25, 2010

\section{ABSTRACT}

\begin{abstract}
A dequate denture hygiene can prevent and treat infection in edentulous patients. They are usually elderly and have difficulty for brushing their teeth. Objective: This study evaluated the efficacy of complete denture biofilm removal using chemical (alkaline peroxide-effervescent tablets), mechanical (ultrasonic) and combined (association of the effervescent and ultrasonic) methods. Material and Methods: Eighty complete denture wearers participated in the experiment for 21 days. They were distributed into 4 groups $(n=20)$ : (1) Brushing with water (Control); (2) Effervescent tablets (Corega Tabs); (3) Ultrasonic device (Ultrasonic Cleaner, model 2840 D); (4) Association of effervescent tablets and ultrasonic device. All groups brushed their dentures with a specific brush (Bitufo) and water, 3 times a day, before applying their treatments. Denture biofilm was collected at baseline and after 21 days. To quantify the biofilm, the internal surfaces of the maxillary complete dentures were stained and photographed at $45^{\circ}$. The photographs were processed and the areas (total internal surface stained with biofilm) quantified (Image Tool 2.02). The percentage of the biofilm was calculated by the ratio between the biofilm area multiplied by 100 and the total area of the internal surface of the maxillary complete denture. Results: The Kruskal-Wallis test was used for comparison among groups followed by the Dunn multiple-comparison test. All tests were performed respecting a significance level of 0.05 . Significant difference was found among the treatments $(K W=21.18 ; P<0.001)$, the mean ranks for the treatments and results for Dunn multiple comparison test were: Control (60.9); Chemical (37.2); Mechanical (35.2) and Combined (29.1). Conclusion: The experimental methods were equally effective regarding the ability to remove biofilm and were superior to the control method (brushing with water). Immersion in alkaline peroxide and ultrasonic vibration can be used as auxiliary agents for cleaning complete dentures.
\end{abstract}

Key words: Denture. Biofilms. Denture cleansers. Peroxides. Ultrasonic. 


\section{INTRODUCTION}

Several studies have mentioned the precarious conditions of oral health of denture wearers ${ }^{14}$. Poor hygiene is associated with the lack of guidance, intrinsic characteristics of dentures and diminished manual dexterity of most denture wearers due to old age $^{20}$.

Poor denture hygiene allows the accumulation of biofilm, which is defined as a dense microbial layer, formed by microorganisms and their metabolites, consisting of more than $10^{11}$ microorganisms per gram of dry weight ${ }^{16}$. When it is properly removed, the result is a reduction in organic material accumulation and proliferation of bacteria and fungi that can cause bad breath, acrylic resin pigmentation and staining, formation of calculus deposits and the development of chronic atrophic candidiasis, also known as denture stomatitis ${ }^{25}$. Sometimes, microorganisms are spread and lung or gastrointestinal infections can also occur ${ }^{17}$. Thus, it can be suggested that adequate denture hygiene can prevent some of the uncomfortable conditions and oral diseases associated with denture wearing.

Denture hygiene methods can be divided into mechanical or chemical procedures ${ }^{20,21}$. Mechanical methods comprise brushing and ultrasonic treatments ${ }^{17}$. Although brushing is the most widespread 12,25 , simple, inexpensive and effective method 20,21 , patients with motor incoordination find it difficult to perform ${ }^{12}$ and there is a possibility of acrylic resin wear ${ }^{9,10}$ and superficial damage to relining materials, therefore it is essential to use adequate brushes and auxiliary agents? ${ }^{7}$.

Ultrasonic devices are mechanical aids generally used by professionals ${ }^{6}$. The mechanical cleansing activity of the device is complemented with the concomitant use of a chemical solution ${ }^{25}$. Ultrasound has two mechanisms of action, the first being the movement of liquid resulting from sound waves transferred to the liquid (vibration), and the second, the collapse of bubbles formed by vibration of the unit ${ }^{22}$.

Chemical methods are classified according to their composition and mechanism, i.e., hypochlorites, peroxides, enzymes, acids, crude drugs and mouth washes for dentures ${ }^{17}$. Immersion of complete dentures in alkaline peroxide is a simple hygiene method. When these peroxides are dissolved in water, they become alkaline hydrogen peroxide, which decomposes and releases small oxygen bubbles with the mechanical action of detaching the biofilm from the denture surface ${ }^{6}$. This type of solution can be used alone or in combination with a mechanical method $17,20,26$.

Clinical experiments show varying results regarding the effectiveness of such agents, demonstrating superiority of the chemical method ${ }^{5,13}$, brushing ${ }^{29}$, or an association of the two methods ${ }^{20}$.

In the literature, the effectiveness of ultrasound is contradictory, as it is attributed to the mechanical action of the device ${ }^{23}$ or to the chemical solutions used $^{18}$. The combination of this method with brushing or with a chemical immersion method has been suggested as an effective alternative for cleaning complete dentures; however, this effectiveness has not been clinically tested. Although the comparison of chemical methods and brushing is relatively common ${ }^{13,20,21}$, the comparison of soaking solutions, ultrasound and their association has not previously been described ${ }^{28}$.

Literature reviews about the efficacy of denture cleansers, dating back to the study of Nikawa ${ }^{17}$ (1999), Shay ${ }^{25}$ (2000) up to the recent study of Souza, et al. ${ }^{28}$ (2009) have shown that there is no consensus about which is the best denture hygiene method, since it is difficult to make comparisons between different studies because of the use of different methodologies to evaluate denture hygiene methods. Therefore, the objective of this study was to evaluate the clinical effectiveness of chemical (sodium perborate-based effervescent tablets), mechanical (ultrasound unit) and combined methods (chemical + mechanical), when associated with brushing, for the removal of complete denture biofilm.

\section{MATERIAL AND METHODS}

\section{Patient selection}

After approval by the institutional Ethics Committee and signature of informed consent forms by the eligible participants, 80 patients aged 4580 years ( 13 men and 47 women) were selected. These individuals needed denture replacements and were under treatment at the Department of Dental Materials and Prosthodontics of the Ribeirão Preto Dental School. The participants presented good general health and were wearing conventional maxillary and mandibular complete dentures. The complete dentures were fabricated from heatactivated acrylic resin, had been in use for periods of time ranging between 5 and 10 years, and received $\geq 1$ scores according to the Additive Index of Ambjørnsen, et al. ${ }^{1}$ (1982).

\section{Hygiene methods and experimental design}

The experimental period lasted 21 days. Before the use of each method, the biofilm was eliminated by brushing with a specific brush for complete dentures (Denture - Condor S.A., São Bento do Sul, SC, Brazil) and liquid soap (JOB Química, Produtos para Limpeza Ltda., Monte Alto, SP, Brazil). During the 21-day period, all participants brushed their dentures 3 times a day. Brushing performed by 
patients ended on the $21^{\text {st }}$ day. In the morning of the $22^{\text {nd }}$ day, they returned to the clinic without having brushed their dentures again and handed them over to the researchers.

The patients were randomly assigned to groups each using one of the following hygiene methods $(n=20)$ :

1) Control:

a) Brushing the dentures 3 times a day, after each meal (breakfast, lunch and dinner) for 2 min using tap water and a specific brush for complete dentures (Bitufo, Itupeva, SP, Brazil); b) Rinsing the oral cavity with running water after brushing; c) Keeping the dentures immersed in water during sleep.

2) Experimental 1:

a) Brushing the dentures 3 times a day, after each meal (breakfast, lunch and dinner) for 2 min using tap water, and a specific brush for complete dentures (Bitufo, Itupeva, SP, Brazil); b) Rinsing the oral cavity with running water after brushing; c) Soaking the dentures in a receptacle with warm water $\left(37^{\circ} \mathrm{C}\right)$ and one effervescent tablet (Corega tabs - Block Drug Company, Inc., USA) for 20 min after dinner; d) Rinsing the dentures before insertion into the oral cavity; e) Keeping the dentures immersed in water during sleep.

3) Experimental 2:

a) Brushing the dentures 3 times a day, after each meal (breakfast, lunch and dinner) for 2 min, with a specific brush for complete dentures (Bitufo, Itupeva, SP, Brazil) and tap water; b) Rinsing the oral cavity with running water after brushing; c) Keeping the dentures immersed in water during sleep; d) At the end of the experimental period (21 days), immersion of the dentures in a sterile beaker containing $250 \mathrm{~mL}$ of sterile water, and ultrasonic vibration (Ultrasonic Cleaner, modelo2840 D Odontobrás Ind. e Com. Equip. Méd. Odont. Ltda, Ribeirão Preto, SP, Brazil) for 15 min, performed by a professional.

4) Experimental 3: combination of methods 2 and 3 .

\section{Percentage of area covered with biofilm}

The internal surfaces of the upper dentures were disclosed by $1 \%$ neutral red solution. The surfaces were then photographed (digital camera: Canon EOS Digital Rebel EF-S 18-55; and flash: Canon MR-14 EX, Canon Inc., Tokyo, Japan) with standard film-object distance and exposure time. The camera was fixed on a stand (CS-4 Copy Stand, Testrite Inst. Co., Inc., Newark, NJ, USA). Photographs were transferred to a computer. Total surface area and areas corresponding to the stained region were measured using image processing software (Image Tool 2.02) (Figure 1). The biofilm percentage was calculated using the ratio between biofilm area multiplied by 100 , and total surface area of the internal denture base $20,24,27$. The researcher who measured biofilm coverage was blind to the group to which the dentures belonged, did not participate in the other experimental phases, such as giving instructions, delivering products to patients, or handling the dentures.

After the use of each method and quantification of the biofilm, it was eliminated by brushing with a specific brush for complete dentures (Denture Condor S.A.,São Bento do Sul, SC, Brazil) and liquid soap (JOB Química, Produtos para Limpeza Ltda., Monte Alto, SP, Brazil).

\section{Data analysis}

The variable "percentage area" was submitted to

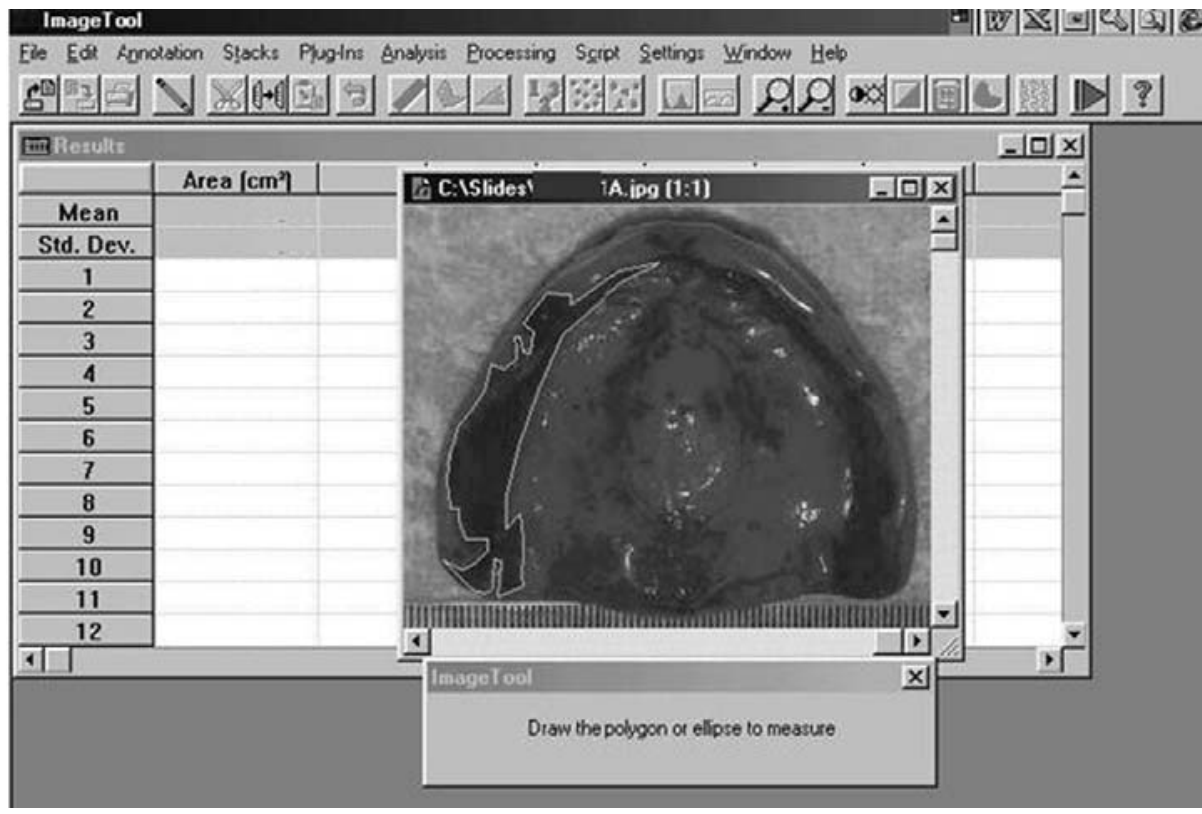

Figure 1- Stained regions were measured using image processing software (Image Tool 2.02) 
Table 1- Percentages of biofilm after the use of each method tested for 21 days

\begin{tabular}{cccc}
\hline Control & $\begin{array}{c}\text { Chemical } \\
\text { (Corega) }\end{array}$ & $\begin{array}{c}\text { Mechanical } \\
\text { (Ultrasound) }\end{array}$ & $\begin{array}{c}\text { Combined } \\
\text { (Corega + Ultrasound) }\end{array}$ \\
\hline 41.10 & 5.48 & 41.64 & 16.07 \\
\hline 29.46 & 21.73 & 7.16 & 2.96 \\
\hline 54.62 & 26.61 & 10.36 & 18.70 \\
\hline 36.78 & 56.36 & 24.69 & 41.80 \\
\hline 27.97 & 14.03 & 0.00 & 12.46 \\
\hline 13.74 & 25.76 & 7.65 & 28.43 \\
\hline 35.17 & 41.98 & 4.28 & 4.55 \\
\hline 15.63 & 13.58 & 13.98 & 27.15 \\
\hline 50.83 & 42.71 & 8.06 & 0.00 \\
\hline 40.40 & 15.21 & 39.92 & 52.53 \\
\hline 43.44 & 7.78 & 38.96 & 8.67 \\
\hline 12.88 & 7.40 & 16.42 & 0.00 \\
\hline 62.20 & 6.66 & 15.97 & 0.50 \\
\hline 24.64 & 24.98 & 12.45 & 0.00 \\
\hline 79.27 & 39.89 & 15.92 & 1.26 \\
18.92 & 3.89 & 7.76 & 1.21 \\
\hline 22.07 & 1.12 & 12.05 & 15.68 \\
88.64 & 1.76 & 10.81 & 18.29 \\
\hline 72.78 & 6.05 & 20.89 & 6.29 \\
\hline 16.52 & 5.18 & 5.53 & 6.14 \\
\hline & & & \\
\hline
\end{tabular}

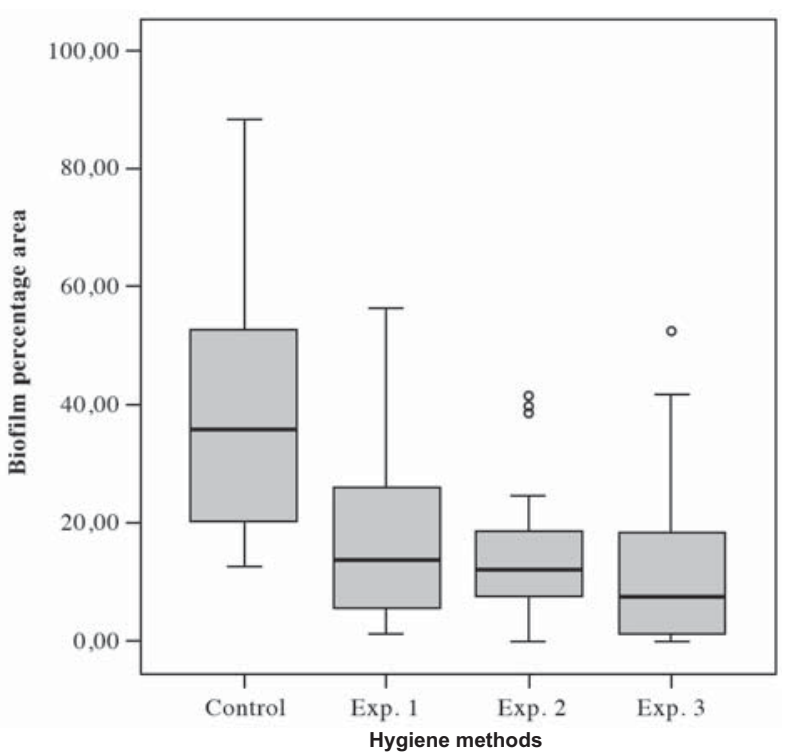

Figure 2- Biofilm coverage area for each group following treatment $($ Exp. $=$ Experimental)

a factor of variation with four levels, representing the tested interventions. The groups presented values with distribution that was not close to normality and had no homogeneous variations (Levene test, $\mathrm{P}=0.035$ ). This called for nonparametric analysis. The Kruskal-Wallis test was
Table 2- Mean ranks for the treatments and results for Dunn multiple comparison test

\begin{tabular}{ccc}
\hline Treatment & Mean rank & Grouping $^{*}$ \\
\hline Control & 60.5 & $\mathrm{~A}$ \\
Chemical & 37.2 & $\mathrm{~B}$ \\
Mechanical & 35.2 & $\mathrm{~B}$ \\
Combined & 29.1 & $\mathrm{~B}$ \\
\hline
\end{tabular}

Identical letters denote no significant differences between the treatments.

used for comparison among groups followed by the Dunn multiple-comparison test. All tests were performed respecting a significance level of 0.05 .

\section{RESULTS}

The results of the biofilm percentage areas after the trial are presented in Table 1 and Figure 2.

The Control treatment appeared to remove less biofilm than the other methods. The experimental methods appeared to be similar for biofilm removal.

The Kruskal-Wallis test found significant difference among the treatments $(\mathrm{KW}=21.18$; $\mathrm{P}<0.001$ ). The experimental methods were similar, whereas the control group was significantly different 
from the former (Table 2). This implies that for denture hygiene, brushing requires auxiliary agents to achieve better results. However, the tested agents - peroxide solution, ultrasound or their combination - attained similar outcomes.

\section{DISCUSSION}

The amount of denture biofilm is associated with the presence of oral lesions ${ }^{14}$. Thus, its quantification can be regarded as a good measurement for denture hygiene outcome. In the present study, the photographic method associated with a computerized method of biofilm quantification was chosen. The first method mentioned above was chosen because quantification by visual inspection ${ }^{1}$ is known to be difficult and the second method mentioned was chosen because it relies on calibration ${ }^{19}$.

Previous analyses by the photographic method, used for comparing total surface areas (internal and external) of complete dentures, showed high correlation coefficients ${ }^{19}$. According to the study of Paranhos and Silva-Lovato ${ }^{19}$ (2004), biofilm quantification performed by photographic and computerized methods, offer objective and accurate results, and should therefore be the methods of choice in clinical experiments for the evaluation of complete denture cleansers. It is important to emphasize that although the computerized method was shown to be reliable for measuring biofilm on complete dentures, it has the important limitation of requiring a longer amount of time spent on making the measurements. Thus, in the present study, the photographic method was associated with the computerized method to evaluate the effectiveness of chemical denture cleansers and an ultrasonic device for biofilm removal from complete dentures.

The mechanical method of performing denture hygiene is very popular among elderly complete denture wearers, however, the immersion of dentures in effervescent alkaline peroxide solutions is also widely used ${ }^{12,25}$. However, lack of access to chemical materials, the cost, and even the patient's lack of adequate information about them limit their use. An alternative method of hygiene is the ultrasonic device, which is considered a fast and effective means of cleaning instrumental devices ${ }^{18}$, and is an important aid in the control of cross contamination. Such devices are commonly found in hospitals, asylums and dental schools ${ }^{25}$.

As in previous studies ${ }^{8,24}$, the results demonstrated that brushing with water favored biofilm accumulation (Figure 2), emphasizing the need to incorporate an auxiliary agent to assist brushing. Microbiologic assays and scanning electron microscopic images have demonstrated that using a denture brush with water is ineffective for removing an unacceptably large proportion of adherent microorganisms ${ }^{25}$. The difference observed between the control and chemical methods (Table 1), indicated that the combination of immersion in the alkaline peroxide solution and brushing improved the effectiveness of hygiene, even within a short period of immersion ( $5 \mathrm{~min}$ ). These results are in agreement with Sheen and Harrison $^{26}$ (2000) and Paranhos, et al. ${ }^{20}$ (2007), who found the chemical methods effective in standardized clinical trials, when compared with control groups using water.

Similarly, there was a significant decrease in the biofilm levels with the use of ultrasound (Figure 2), demonstrating the effectiveness of the method, even when it was used only once during the experiment. This effectiveness can be attributed exclusively to the ultrasonic cavitation, as no chemical substance was incorporated to the apparatus.

The statistical equality between the chemical and mechanical methods indicated that the use of ultrasound alone achieved the same results as those obtained by using the tablets daily. Previous studies demonstrated the superiority of ultrasound, in comparison with the alkaline peroxide solutions, even when used with water ${ }^{11,18,23}$, however, the ultrasound unit was used daily.

Although the use of the combined method provided greater reduction in biofilm levels, the means obtained (Table 1 ) were statistically equal to those of the groups in which the methods were used alone. Paranhos, et al. ${ }^{20}$ (2007) found greater effectiveness with the combined method; however, for the mechanical method, specific brushes and toothpastes were used to clean the complete dentures, products that proved to be effective in removing biofilm 21,24,27.

As regards the mechanical method, experiments incorporating antiseptic solutions such as sodium hypochlorite are important, since studies have shown that the effectiveness of these solutions is superior when compared with peroxides ${ }^{13,15}$. Microbiological comparative studies have concluded that effective hygiene is obtained by the combination of chemical (immersion) and mechanical (brushing) methods ${ }^{5}$.

This way, microbiological experiments that evaluate the effectiveness of the combination of the immersion with the ultrasound should be conducted in future studies. With regard to the chemical method, studies should be conducted using the products in prolonged periods of immersion, since previous studies indicated that short periods are less effective in comparison with prolonged periods of immersion (overnight) ${ }^{4}$. Future comparisons conducted in a clinical trial design could assess the use of different chemicals within the ultrasonic device, i.e., surfactants or antimicrobial agents, 
since an important limitation of this study was that the peroxides were not used as conducting fluid in the ultrasonic device.

\section{CONCLUSION}

The three methods used (chemical, mechanical and combined) were equally effective with respect to the ability to remove biofilm and were superior to the established control method (brushing with water), contributing to the maintenance of oral health care of the complete denture wearers.

\section{ACKNOWLEDGEMENTS}

\author{
The State of São Paulo Research Foundation \\ (FAPESP) for financial support.
}

\section{REFERENCES}

1- Ambjørnsen E, Rise J. The effect of verbal information and demonstration on denture hygiene in elderly people. Acta Odontol Scand. 1985;43:19-24.

2- Ambjørnsen E, Valderhaug J, Norheim PW, Fløystrand F. Assessment of an additive index for plaque accumulation on complete maxillary dentures. Acta Odontol Scand. 1982;40:203-8. 3- Andrucioli MCD, Macedo LD, Panzeri H, Lara EHG, Paranhos HF. Comparison of two cleansing pastes for the removal of biofilm from dentures and palatal lesions in patients with atrophic chronic candidiasis. Braz Dent J. 2004;15:220-4.

4- Augsburger RH, Elahi JM. Evaluation of seven proprietary denture cleansers. J Prosthet Dent. 1982;47:356-9.

5- Chan ECS, Iugovaz I, Siboo R, Bilyk M, Barolet R, Amsel R, et al. Comparison of two popular methods for removal and killing of bacteria from dentures. J Can Dent Assoc. 1991;57:937-9.

6- Council on dental materials, instruments and equipment. Denture cleansers. J Am Dent Assoc. 1983;106:77-9.

7- Dyer D, MacDonald E, Newcombe RG, Scratcher C, Ley F, Addy M. Abrasion and stain removal by different manual toothbrushes and brush actions: studies in vitro. J Clin Periodontol. 2001;28:121-7.

8- Fernandes RAG, Lovato-Silva CH, Paranhos HF, Ito IY. Efficacy of three denture brushes on biofilm removal from complete dentures. J Appl Oral Sci. 2007;15:39-43.

9- Freitas KM, Paranhos HF. Weight loss of five commercially available denture teeth after toothbrushing with three different dentifrices. J Appl Oral Sci. 2006;14:242-6.

10- Freitas-Pontes $\mathrm{KM}$, Silva-Lovato $\mathrm{CH}$, Paranhos HFO. Weight loss of four commercially available heat-polymerized acrylic resins after toothbrushing with three different dentifrices. J Appl Oral Sci. 2009;17:116-21.

11- Gwinnett AJ, Caputo L. The effectiveness of ultrasonic denture cleaning: a scanning electron microscope study. J Prosthet Dent. $1983 ; 50: 20-5$.
12- Jagger DC, Harrison A. Denture cleansing-the best approach. Br Dent J. 1995;178:413-7.

13- Kulak Y, Arikan A, Albak S, Okar I, Kazazoğlu E. Scanning electron microscopic examination of different cleaners: surface contaminant removal from dentures. J Oral Rehabil. 1997;24:20915.

14- Marchini L, Vieira PC, Bossan TP, Montenegro FL, Cunha VP. Self-reported oral hygiene habits among institutionalized elderly and their relationship to the condition of oral tissues in Taubaté, Brazil. Gerodontology. 2006;23:33-7.

15- Moore TC, Smith DE, Kenny GE. Sanitization of dentures by several denture hygiene methods. J Prosthet Dent. 1984;52:15863.

16- Nikawa H, Hamada T, Yamamoto T. Denture plaque - past and recent concerns. J Dent. 1998;26:299-304.

17- Nikawa $\mathrm{H}$, Hamada T, Yamashiro $\mathrm{H}$, Kumagai H. A review of in vitro and in vivo methods to evaluate the efficacy of denture cleansers. Int J Prosthodont. 1999;12:153-9.

18 - Palenik $\mathrm{CJ}$, Miller $\mathrm{CH}$. In vitro testing of three denture-cleaning systems. J Prosthet Dent. 1984;51:751-4.

19- Paranhos HF, Silva CHL. Comparative study of methods for the quantification of biofilm on complete dentures. Braz Oral Res. 2004;18:215-23.

20- Paranhos HF, Silva-Lovato $\mathrm{CH}$, Souza RF, Cruz PC, Freitas $K M$, Peracini A. Effects of mechanical and chemical methods on denture biofilm accumulation. J Oral Rehabil. 2007;34:606-12. 21- Paranhos HF, Silva-Lovato CH, Venezian GC, Macedo LD, Souza RF. Distribution of biofilm on internal and external surfaces of upper complete dentures: the effect of hygiene instruction. Gerodontology. 2007;24:162-8.

22- Pitt WG, Ross SA. Ultrasound increases the rate of bacterial cell growth. Biotechnol Prog. 2003;19:1038-44.

23- Raab FJ, Taylor CA, Bucher JA, Mann BL. Scanning electron microscopic examination of ultrasonic and effervescent methods of surface contaminant removal from complete dentures. J Prosthet Dent. 1991;65:255-8.

24- Salles AES, Macedo LD, Fernandes RAG, Silva-Lovato $C H$, Paranhos HF. Comparative analysis of biofilm levels in complete upper and lower dentures after brushing associated with specific denture paste and neutral soap. Gerodontology. 2007;24:217-23. 25- Shay K. Denture hygiene: a review and update. J Contemp Dent Pract. 2000;1:1-8.

26- Sheen SR, Harrison A. Assessment of plaque prevention on dentures using an experimental cleanser. J Prosthet Dent. 2000;84:594-601.

27- Silva CHL, Paranhos HF. Efficacy of biofilm disclosing agent and of three brushes in the control of complete denture cleansing. J Appl Oral Sci. 2006;14:454-9.

28- Souza RF, Freitas Oliveira Paranhos $H$, Lovato da Silva $\mathrm{CH}$, Abu-Naba'a L, Fedorowicz Z, Gurgan CA. Interventions for cleaning dentures in adults. Cochrane Database Syst Rev. 2009; 7:CD007395.

29- Tarbet WJ, Axelrod S, Minkoff S, Fratarcangelo PA. Denture cleansing: a comparison of two methods. J Prosthet Dent. 1984;51:322-5. 